\title{
Micromegas as a large microstrip detector for the COMPASS experiment
}

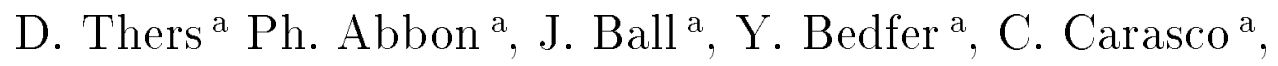 \\ E. Delagnes ${ }^{\text {a }}$, D. Durand ${ }^{\text {a }}$, J.-C. Faivre ${ }^{\text {a }}$, H. Fonvieille ${ }^{\text {, }}$, \\ A. Giganon ${ }^{\text {a }, ~ F . ~ K u n n e ~}{ }^{\mathrm{a}, 1}$, J.-M. Le Goff ${ }^{\text {a }}$, F. Lehar ${ }^{\text {a }}$, \\ A. Magnon ${ }^{\text {a }}$, D. Neyret ${ }^{a}$, E. Pasquetto ${ }^{\text {a }}$,H. Pereira ${ }^{\text {a }}$, \\ S. Platchkov ${ }^{\text {a }}$, E. Poisson ${ }^{\text {a }}$ and Ph. Rebourgeard ${ }^{\text {a }}$

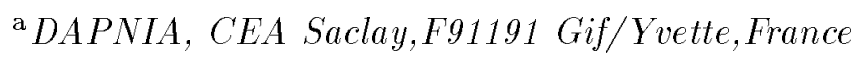 \\ ${ }^{\mathrm{b}}$ LPC IN2P3/CNRS, Univ.Blaise Pascal Clermont II, F69177 Aubiere, France
}

\begin{abstract}
Recent results on the gazeous microstrip detector Micromegas which will be used to track particles in the COMPASS experiment at CERN are presented. Developments bearing on its mechanical and electrical design, associated readout electronics and gas mixture were carried out. A particular attention was paid to the discharge phenomenom which affects this type of microstrip detector. The adequacy of the options finally retained, especially the SFE16 readout and the use of a $\mathrm{Ne}-\mathrm{C}_{2} \mathrm{H}_{6}-$ $\mathrm{CF}_{4}$ gas mixture was demonstrated in a set of beam tests performed on a $26 \times 36 \mathrm{~cm}^{2}$ prototype. Operating at a gain of $\sim 6400$, full efficiency is reached along with a spatial resolution of $50 \mu \mathrm{m}$ and a timing accuraccy of $8.5 \mathrm{~ns}$. When operating with the muon beam, there is less than one discharge per SPS spill. Via a decoupling of the strips through individual capacitors their impact is somewhat reduced. For the studied prototype, discharges generate a dead time on the full detector of $\sim 3 \mathrm{~ms}$, affecting marginally the detection efficiency given their rate. The probability of discharge is found to vary with the mean value of the gas mixture atomic number: gases with low atomic numbers produce less discharges. In view of these results, the commissioning of Micromegas for COMPASS is foreseen in 2000.
\end{abstract}

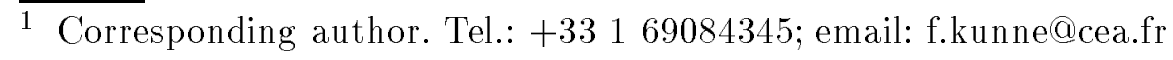




\section{Introduction}

The COMPASS experiment at CERN [1] has been set up to address a number of physics objectives, in particular the measurement of the gluon spin contribution to the nucleon spin, through photon gluon fusion $(\gamma g \rightarrow q \bar{q})$ in the scattering of longitudinally polarized muons on longitudinally polarized target nucleons. Such a measurement calls for a high integrated luminosity combined with a large acceptance and a high precision tracking. In the fixed target realm, the conditions under which the tracking system operates vary strongly, when moving both along the beam and transverse to it. The most stringent requirements concern the section of the spectrometer downstream of the target and upstream of the analyzing magnets, where the detectors sense the full spectrum of particles produced in the target since the low energy component is not yet swept by the magnetic field. When moving in the transverse direction, the particles rate is highest in the beam axis and decreases when moving out of the axis. Over the large acceptance of COMPASS this translates into a variety of constraints, which is coped with by subdividing the tracking system into a set of nested detectors of increasing rate capabilities. The center-most detectors, called Very Small Area Trackers (VSAT), cover the limited area of the beam region. Scintillating fibers are foreseen for that task. The intermediate region and the outermost region are covered by the Small Angle Trackers (SAT), and Large Angle Trackers (LAT) respectively. For the LAT the reduced flux allows the use of drift detectors. We report here on the ability of Micromegas gaseous microstrip detectors [2][3] equipped with a dedicated preamplifier-discriminator circuit, the SFE16 [4], to fulfill the demands for the SAT in the section upstream of the analyzing magnet.

We foresee 12 planes of Micromegas of $40 \times 40 \mathrm{~cm}^{2}$, excluding a $5 \mathrm{~cm}$ diameter central dead zone, and $\sim 400 \mu \mathrm{m}$ pitch. In addition to the above mentioned rate constraints the experiment sets also constraints on the position accuracy of the detectors and their total mass, which determine the magnitude of the multiscattering. The requirements that result from this set of constraints and options are the following:

- Spatial resolution better than $100 \mu m$.

- Rate capability, for a typical beam of $\sim 10^{8} \mathrm{~s}^{-1}$ muons of $200 \mathrm{GeV}$ energy on a $1.3 \mathrm{~m}$ DLi target: $\sim 20 \mathrm{MHz}$ integrated and $\sim 90 \mathrm{kHz}$ maximum rate per strip.

- Low mass

Another important constraint is set by the fact that Micromegas, like other micro-strip detectors is subject to discharges. We have shown that hadrons produce more discharges than electrons or muons. However, in the case of a muon beam, secondary hadrons are produced in the target, and some hadrons 
may also be present in the beam halo. They are most likely responsible for the discharges observed with the high energy muon beam. We give here the performances of Micromegas prototypes tested successively with radioactive sources, in a high energy hadron beam at CERN, and finally in the M2 muon beam at CERN in a COMPASS-like environment.

\section{The Micromegas detector}

\subsection{Principle}

Micromegas is a gaseous detector using a parallel planes electrode structure and microstrips [2]. The special feature of this detector is the use of a micromesh which separates the detector into two regions, a $2.5 \mathrm{~mm}$ thick conversion (or drift) gap and a $100 \mu \mathrm{m}$ amplification gap. The charges generated by the ionizing particles are collected in the conversion gap and drift in a moderate electrical field of $1 \mathrm{kVcm}^{-1}$. The electrons cross the micromesh and reach the amplification gap where the electric field of $50 \mathrm{kV} / \mathrm{cm}$ produces an avalanche resulting in a large number of electron/ion pairs. The field configuration prevents these ions from drifting back into the conversion gap by stopping most of them when they cross the mesh. Consequently the ions drift over a maximum distance of $100 \mu \mathrm{m}$. The width of the signal induced on the strips cannot exceed the drift time over that distance which is $\approx 100 \mathrm{~ns}$. The fast evacuation of positive ions combined with the reduced transverse scattering of the electrons (over a few hundred microns) and the high granularity of the detector provide a high rate capability.

\section{D Description}

The prototypes tested for the COMPASS experiment differ significantly in their size and mechanical structure from the Micromegas detectors previously described [2]. The active area is $260 \mathrm{~mm} \times 360 \mathrm{~mm}$ and the micromesh is stretched and glued on a removable frame which facilitates the detector repair. In this version, Micromegas consists of two main components: the substrate and the mesh frame.

The substrate. Anode copper strips, $7 \mu \mathrm{m}$ thick, $250 \mu \mathrm{m}$ wide with a pitch of $317 \mu \mathrm{m}$ are printed on a $400 \mu \mathrm{m}$ thick fiber epoxy substrate. To improve the rigidity, a $5 \mathrm{~mm}$ honeycomb glued to a $250 \mu \mathrm{m}$ carbon epoxy sheet is then glued to the fiber epoxy substrate. Cylindrical insulators made of photoresist, $100 \mu \mathrm{m}$ thick and $150 \mu \mathrm{m}$ in diameter are fixed to the anode strips by standard 
printed circuit technique. They define very accurately the amplification gap of the Micromegas detector.

The mesh frame. The drift mesh and the inner micromesh are both glued on a glass fiber frame with a $7 \mathrm{~mm} \times 2.45 \mathrm{~mm}$ section. The conversion gap or drift space is defined by the $2.45 \mathrm{~mm}$ thickness of the frame. The amplification micromesh is an electroformed nickel grid, $4 \mu \mathrm{m}$ thick having $37 \mu \mathrm{m}$ square holes every $50 \mu \mathrm{m}$. The drift mesh is similar but has a $100 \mu \mathrm{m}$ pitch. The novel concept of drift electrode fabricated out of a mesh improves the flow of the gas mixture.

A thin kapton foil $\left(5 \times 5 \mathrm{~cm}^{2}\right)$ was introduced in the amplification gap to neutralize the amplification in the centre of the detector. This area intercepts the high intensity muon beam which produces a too large occupancy in the corresponding strips.

The gas flows (2.5 liters/hour) through the whole chamber within a volume defined by the substrate and the mylar external window (1.2 liters in total). The total gas volume is renewed twice per hour.

To polarize the detector, separate voltages are applied to the drift and the mesh electrodes. They are both connected to negative supplies through R-C$\mathrm{R}$ filters. We have used $500 \mathrm{~K} \Omega-10 \mathrm{nF}-500 \mathrm{k} \Omega$ for the drift electrode and 500 $\mathrm{k} \Omega-10 \mathrm{nF}-100 \Omega$ for the micromesh. The anode strips are grounded via 10 $\mathrm{M} \Omega$ resistors and coupled to the preamplifiers through a $100 \mathrm{pF}$ capacitance.

The detector mass is less than $500 \mathrm{~g}$ and the active area represents $\sim 0.45 \%$ radiation length. We foresee to use a thinner epoxy substrate which will improve this figure.

\section{Front end electronics}

\subsection{Constraints for the front end electronics}

Several constraints have guided the choice of the parameters for the design of the front-end electronics. Measurements with Micromegas equipped with fast amplifiers LeCroy MQS104A (15 ns rise time leading to a $70 \mathrm{~ns}$ minimum signal width) were performed initially in the high intensity M2 muon beam, in the COMPASS beam region. With $\sim 10^{8}$ muons per second, the rate per strip was a few $\mathrm{MHz}$ with the detector centered on the beam and the preamplifier shaping is still too large to ensure an acceptable circuit occupancy. Moreover, the MQS104 has a noise versus capacitance figure $\left(2000 \mathrm{e}^{-}+50 \mathrm{e}^{-} / \mathrm{pF}\right)$ such 
that detection efficiencies nearing $100 \%$ can only be reached at gains beyond $2.10^{4}$. At such gain values, it was shown [5] that Micromegas suffered from frequent discharges. In order to reduce the discharge probability, the SFE16 readout circuit was developed to provide a $\sim 100 \%$ detection efficiency at gains not exceeding few thousands. The longer shaping time, compared to the MQS104 resulted in an even higher occupancy in the beam region. We therefore restricted the use of Micromegas in the COMPASS SAT region, avoiding the $2.5 \mathrm{~cm}$ radius central area. The maximum flux density is of $270 \mathrm{kHz} . \mathrm{cm}^{-2}$ and the maximum rate per strip is of $\sim 90 \mathrm{kHz}$, leading to an occupancy below $5 \%$ for the hottest part.

\section{D The SFE16 preamplifier chip}

The SFE16 preamplifier comparator is described in detail in [4]. We recall here only its main characteristics and performances. Each unit includes 16 front-end channels. The shaper has been designed with a peaking time of 85 ns, providing the best match with input signals of $100 \mathrm{~ns}$ width. The response of the SFE16 preamplifier to a Dirac impulse of $4 \mathrm{fC}$ is a $160 \mathrm{~ns}$ wide signal. When connected to a $26 \mathrm{~cm}$ long strip, we obtain $\sigma_{\text {noise }}=825 \mathrm{e}^{-}$. The channelto-channel baseline dispersion combined with the gain dispersion causes an artificial spread of $750 \mathrm{e}^{-}$in the threshold values. The threshold is common to all 16 channels, and is set at $5240 \mathrm{e}^{-}$in order to ensure a noise frequency below $1 \mathrm{kHz}$ on every channel. This threshold value corresponds to $6.3 \sigma_{\text {noise }}$, a rather high value partly due to the baseline dispersion.

\subsection{Time over threshold measurement}

The read-out involves only the recording of the times $T_{\text {lead }}$ and $T_{\text {trail }}$, at which the leading edge and the trailing edge of the analog signal cross the discriminator threshold (logical read-out). The Time Over Threshold (TOT) of the signal is calculated from these values : TOT $=T_{\text {lead }}-T_{\text {trail }}$. The final value of the time of the signal $T_{\text {mean }}$ is obtained from a combination of $T_{\text {lead }}$ and $T_{\text {trail }}$ with coefficients depending on the TOT value.

The various timing distributions obtained from 64 channels of a Micromegas filled with a $\mathrm{Ne}-\mathrm{C}_{2} \mathrm{H}_{6}-\mathrm{CF}_{4}$ gas mixture, and operating at a gain of 3800 (efficiency $98 \%$ ) in a $15 \mathrm{kHz}$ hadron beam are shown in Fig.1. The full timing distribution covers $160 \mathrm{~ns}$ for the leading edge $T_{\text {lead }}$, and more than $200 \mathrm{~ns}$ for the trailing edge $T_{\text {trail }}$. It is reduced to only $50 \mathrm{~ns}(\sigma=8.5 \mathrm{~ns})$ for the $T_{\text {mean }}$ value which is not affected by the time walk.

We have measured a significant correlation between the TOT value and the 

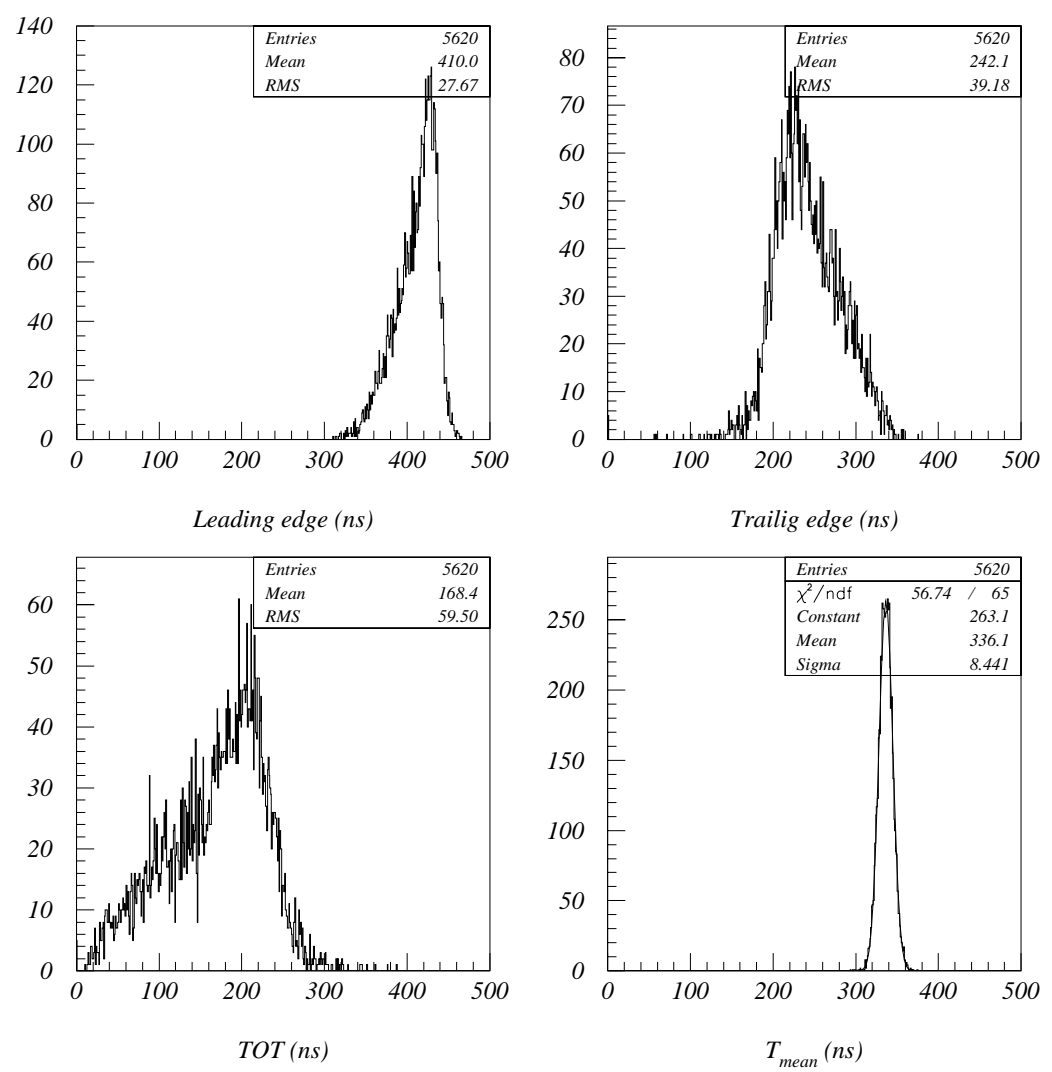

Fig. 1. Timing distributions for 64 channels of the Micromegas chamber equipped with the SFE16 preamp.: leading edge, trailing edge, TOT=trailing-leading, and mean time $T_{\text {mean }}$ obtained as a combination of the leading and trailing edge times; gas: $N e-C_{2} H_{6}-C_{4}(79-11-10)$; beam $10 \mathrm{GeV} \pi$ at $15 \mathrm{kHz}$.

amplitude of the analog signal. The peak at $200 \mathrm{~ns}$ in the distribution of TOT corresponds to signals with large amplitudes for which the detector is fully efficient; the presence of signals with smaller TOT, i.e. small amplitudes, prevents to operate at lower gains. The position of the hit is calculated from the barycentre of the positions of hit strips, weighted by the TOT value. This procedure improves the spatial resolution from 70 to $50 \mu \mathrm{m}$.

\section{Performances for various gas mixtures}

\subsection{Gas mixtures for Micromegas}

Extensive studies of the efficiency, and of the timing and spatial resolutions of the Micromegas detectors filled with various gas mixtures have been performed 
using a ${ }^{90} \mathrm{Sr}$ source. They aimed at determining an optimum gas mixture which guarantees a full detection efficiency for the detector running with its final front-end electronics, at a gain compatible with a negligible rate of discharges when used as a COMPASS tracker. Additional goals were also obtaining a position resolution of $100 \mu \mathrm{m}$ (or better) and a timing accuracy in the $10 \mathrm{~ns}$ range. Obtaining small values for the TOT was also important to minimize the occupancy.

Initial tests were performed using the LeCroy MQS104A preamplifier described above associated with both digital and analog read-outs. Interesting features were found, thanks to the short shaping time of that circuit[3]. The output signal displays an asymmetric shape with a sharp rise over 20-40 ns, and a tail of 40-150 ns which results from the drift of ions in the amplification gap. Gas with a lower ion drift velocity lead to a more asymmetric shape. Measurements performed for several binary gas mixtures, show that the TOT value depends on the mass of the quencher gas (and of the buffer gas) rather than on the proportion. At full efficiency, mean TOT values of 80,100 and $170 \mathrm{~ns}$ are obtained with $C_{2} H_{6}, i C_{4} H_{10}$ (isobutane) and $C_{6} H_{12}$ as quencher (in Argon), almost independently of the proportion of quencher. With $\mathrm{C}_{2} \mathrm{H}_{6}$ in Neon, the TOT is reduced to $75 \mathrm{~ns}$. In general, the narrowest signals are obtained with the lightest buffer and quencher gases.

It was also shown that adding $C F_{4}$ to the binary gas mixture tends to reduce the TOT by a reduction of the rise time. This effect is understood as resulting from the increase in the drift velocity of primary electrons. With the LeCroy MQS104 amplifier, a TOT of $70 \mathrm{~ns}$ is measured with the triple mixture $\mathrm{Ne}-$ $\mathrm{C}_{2} \mathrm{H}_{6}-\mathrm{CF}_{4}$.

Since we decided to equip Micromegas with the SFE16 instead of the MQS104 and because of the significant differences in characteristics like the noise figure and the shaping time, it was not possible to extrapolate the MQS104 results to the new preamplifier. The performances of Micromegas equipped with the SFE16 and filled with similar gas mixtures had to be established again, using both a ${ }^{90} S r$ source and a high energy hadron beam at CERN. The main results are summarized here.

\subsection{Gain measurements}

The amplification factor or gain of the detector is obtained from the charge induced on the mesh when a $5.9 \mathrm{keV}$ photon from a ${ }^{55} \mathrm{Fe}$ source is absorbed in the detector gas. When measuring the gain, the strips have to be electrically grounded. Amplification by an ORTEC charge preamplifier-amplifier results in an output voltage $V_{\text {out }}^{F e}$. For this measurement we used the ORTEC 142B 
preamplifier stage, well adapted to the high capacitance of the detector mesh. To minimize the noise, the final integrating time was set at $1 \mu \mathrm{s}$. The voltage $V_{\text {out }}^{F e}$ is converted into the charge $Q_{i n}^{F e}$ resulting from the photon detection using a calibration of the ORTEC system. The gain $G$ is derived from this quantity using the relation $G=Q_{i n}^{F e} /\left(n_{p}^{F e} \times e\right)$ where $n_{p}^{F e}$ is the number of primary electrons produced by the $5.9 \mathrm{keV}$ photon absorption and $e$ is the electron charge. The quantity $n_{p}^{F e}$ depends on the ionization potential of the gas mixture known for pure gases[6]. The value of $G$ is measured with an uncertainty of $\sim 15 \%$ originating mainly from the error on the determination of $n_{p}^{F e}$ and a measurement uncertainty from the noise on the mesh signal. Results for the gain versus the mesh voltage $V_{\text {mesh }}$ are shown in Fig. 2 using Argon and Neon as buffer gas. For $A r-i C_{4} H_{10}$ mixtures, a given value of $G$ is obtained at higher $V_{\text {mesh }}$ when adding more quencher. A similar, although reduced effect is observed using $\mathrm{Ne}-\mathrm{C}_{2} \mathrm{H}_{6}$ mixtures. However, large variations in $V_{\text {mesh }}$ are again observed if one adds $\mathrm{CF}_{4}$ to the $\mathrm{Ne}-\mathrm{C}_{2} \mathrm{H}_{6}$ mixture. We have observed that with a sizable percentage of $C F_{4},(5$ to $10 \%)$ the gain is not much sensitive to the percentage of quencher for values between 3 and 15 $\%$. However, a minimal amount of quencher $(2-3 \%)$ is necessary in order to guarantee a stable operation of the detector.

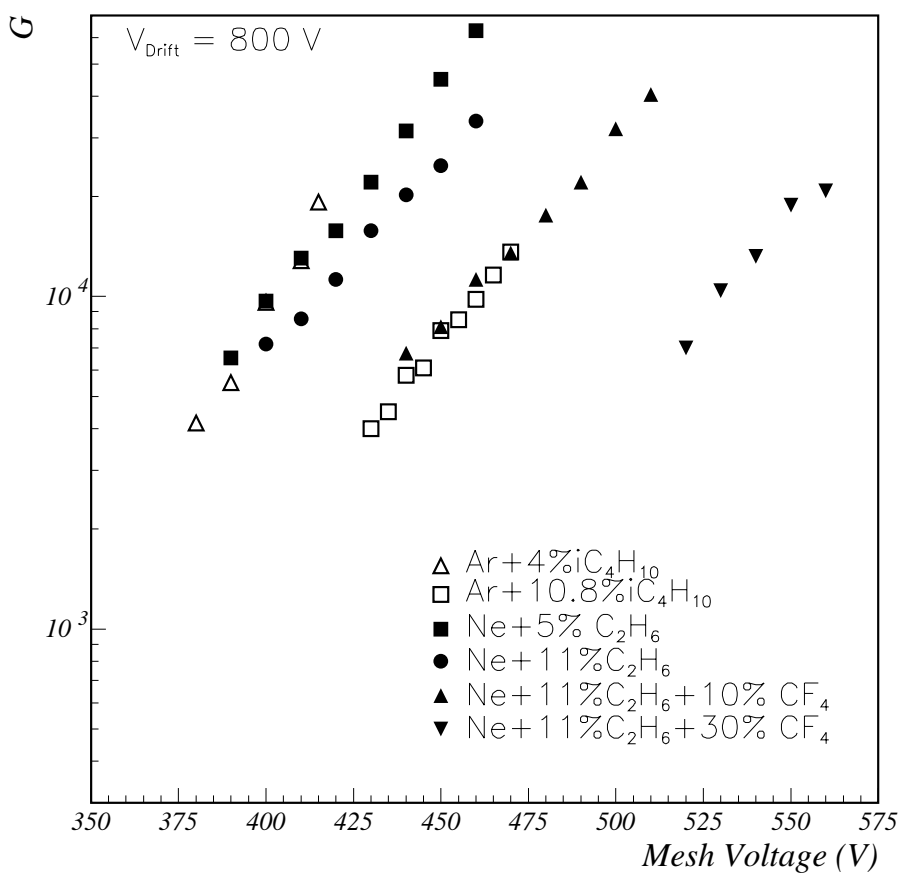

Fig. 2. Gain versus mesh voltage for various gas mixtures. 


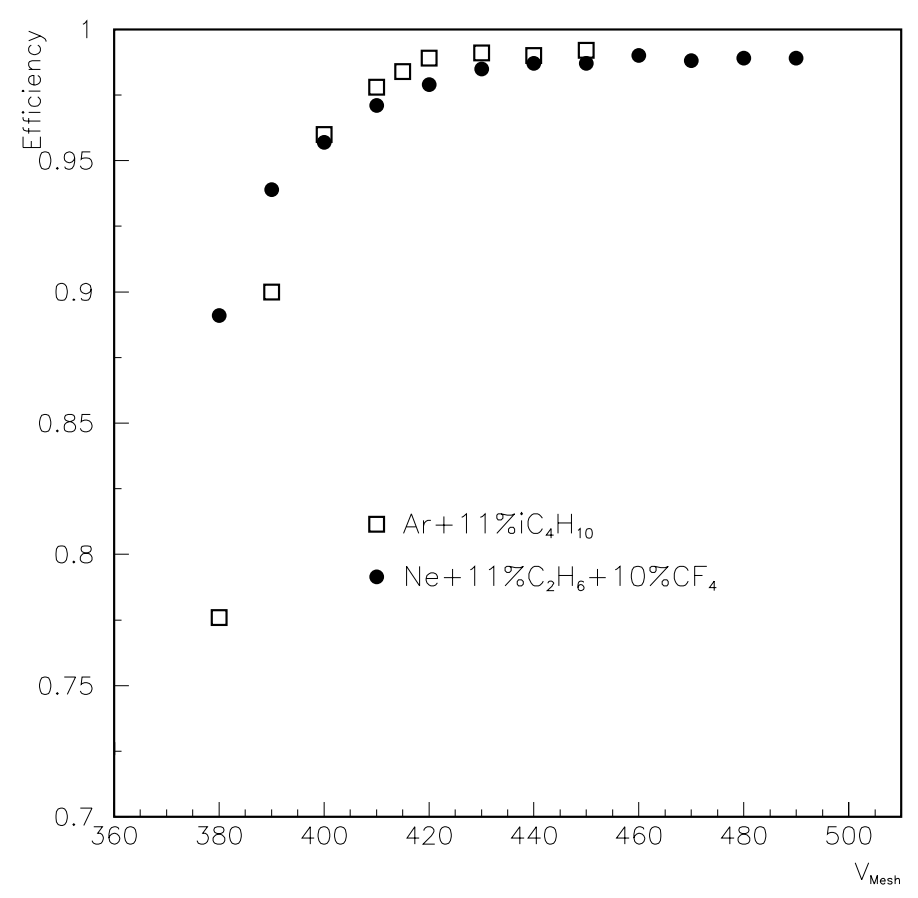

Fig. 3. Efficiency versus mesh voltage for two gas mixtures.

\subsection{Efficiency, time resolution and spatial resolution for various gas mixtures}

The measurements were done using the CERN PS beam line T9, which provides a mixed beam, mostly hadrons of maximum energy around $15 \mathrm{GeV}$. The spill length was $300 \mathrm{~ms}$; we selected energies between 10 and $15 \mathrm{GeV}$ and the total flux of particles was between 15 and $50 \mathrm{kHz}$. The large Micromegas detector described above, equipped with 4 SFE16 (64 horizontal channels) was part of a telescope including 3 other Micromegas of smaller size $\left(15 \times 15 \mathrm{~cm}^{2}\right)$ equipped with MQS104 fast electronics (each with 64 horizontal channels). The detectors were spaced out by $\sim 20 \mathrm{~cm}$ each, and the contribution of multiple Coulomb scattering was negligible at these energies. The acquisition of the detectors was triggered by a coincidence of two small scintillators defining a $1 \times 2 \mathrm{~cm}^{2}$ active area.

Several gas mixtures were studied: two mixtures based on Argon with a proportion of $4 \%$ and $11 \%$ of $i C_{4} H_{10}$ as quencher, two mixtures based on Neon with a proportion of $5 \%$ and $11 \%$ of $C_{2} H_{6}$ as quencher and two triple mixtures based on Neon with $11 \%$ of $\mathrm{C}_{2} \mathrm{H}_{6}$ and $10 \%$ or $30 \%$ of $C F_{4}$.

Results for the detector efficiency are shown in Fig. 3 as a function of the mesh voltage for the two gas mixtures $\mathrm{Ar}-i \mathrm{C}_{4} H_{10}$ (89-11) and $\mathrm{Ne}-\mathrm{C}_{2} \mathrm{H}_{6}-\mathrm{CF}_{4}$ (79-11-10), with the discriminator threshold set at $5240 e^{-}\left(6.3 \sigma_{\text {noise }}\right)$. 
An efficiency of $99 \%$ is reached at mesh voltage values of $\approx 425$ and $\approx 440$ $\mathrm{V}$ corresponding to gain values of $\approx 3700$ and $\approx 6400$ for the Argon and the Neon mixtures respectively. The ionization in Neon leads to a smaller number of primary electrons $n_{e p}$ compared to Argon. Since the total charge $G \times n_{e p}$ is a relevant quantity when dealing with efficiency, we expect, as observed, to operate at higher gain for Neon compared to Argon. A confortable efficiency plateau is obtained with the two mixtures. With the Neon mixture, it extends over more than 50 volts. The main objective of the new chip design was to match the properties of Micromegas to reach high detection efficiencies at gain values of few thousands. The results reported here show that this objective has been met.

The presence of discharges leads us to operate Micromegas at the lowest possible gain, compatible with full efficiency. We choose to quote for each gas mixture, the detector performances at the start up of the efficiency plateau ( $99 \%$ efficiency) where the gain is defined as $G_{0}$. Table 1 shows, at this operating point and for several gas mixtures, the value of the amplification gain $G_{0}$, the mean cluster size $c l_{s z}$ which represents the mean number of adjacent hit strips, the Time Over Threshold (TOT $=T_{\text {lead }}-T_{\text {trail }}$ ) and the position and timing resolutions.

For each gas mixture, the mean number of primary electrons $n_{e p}$ created by a minimum ionizing particle crossing the $2.5 \mathrm{~mm}$ thick drift space, is estimated starting from the number given in ref. [6] for pure gases. The $n_{e p}$ values given in table 1 are corrected for relativistic effects. In the case of the $\mathrm{Ar}-i \mathrm{C}_{4} \mathrm{H}_{10}$ (89-11) and $\mathrm{Ne}-\mathrm{C}_{2} \mathrm{H}_{6}-\mathrm{CF}_{4}$ (79-11-10) mixtures, our measurements were performed with a $10 \mathrm{GeV}$ pion beam, and the $n_{e p}$ values are multiplied by 1.4 . For the four other gas mixtures, the beam was composed of $10 \mathrm{GeV}$ protons and pions, and the correction factor was estimated to 1.16 .

Several features of Table 1 deserve to be outlined:

(1) As expected, for all mixtures, the full efficiency is reached at rather moderate gains ( $G_{0} \approx$ a few thouthands).

(2) The width of the signal (TOT measurement) results from the contributions of the SFE16 shaping time and of the ion drift time. For the $\mathrm{Ne}-\mathrm{C}_{2}-\mathrm{H}_{6}$ mixtures, the TOT is largely dominated by the shaping time of the SFE16. For slower mixtures based on $\mathrm{Ar}-i \mathrm{C}_{4} H_{10}$, the TOT is higher.

(3) The position resolution is related to the mean cluster size $c l_{s z}$. The best resolutions are obtained with the smallest mean cluster sizes. A significant reduction of $c l_{s z}$ occurs when adding $C F_{4}$.

(4) The timing resolution depends on the electron drift time as well as on the internal jitter of the amplifier. It improves when adding quencher. As for the cluster size, a significant improvement by a factor of $\sim 2$ is seen 
Table 1

Performances of a Micromegas detector, at full efficiency for various gas mixtures; read out electronics : SFE16; beam conditions: $10 \mathrm{GeV} \pi$ or $\mathrm{p}$.

\begin{tabular}{|l|l|l|l|l|l|l|}
\hline Gas & $\mathrm{Ar}_{-1 C_{4} \mathrm{H}_{10}}$ & $\mathrm{Ar}_{-1 \mathrm{C}_{4} \mathrm{H}_{10}}$ & $\mathrm{Ne}-\mathrm{C}_{2} \mathrm{H}_{6}$ & $\mathrm{Ne}-\mathrm{C}_{2} \mathrm{H}_{6}$ & $\mathrm{NeC}_{2} \mathrm{H}_{6} \mathrm{CF}_{4}$ & $\mathrm{NeC}_{2} \mathrm{H}_{6} \mathrm{CF}_{4}$ \\
$\%$ & $96-4$ & $89-11$ & $95-5$ & $89-11$ & $79-11-10$ & $59-11-30$ \\
$n_{e p}$ & 28.4 & 36.9 & 13.5 & 14.6 & 19.7 & 19.6 \\
\hline$G_{0}$ & 4100 & 3700 & 14500 & 14000 & 6400 & 7400 \\
cluster size $c l_{s z}$ & 3.0 & 2.4 & 3.4 & 2.9 & 2.1 & 2.2 \\
TOT (ns) & 210 & 195 & 187 & 182 & 171 & 179 \\
RESOLUTIONS: & & & & & & \\
Position $(\mu \mathrm{m})$ & 70 & 62 & 80 & 80 & 50 & 50 \\
Timing $(\mathrm{ns})$ & 17.1 & 12.3 & 17.9 & 16.5 & 8.8 & 8.6 \\
\hline
\end{tabular}

when adding $10 \% C F_{4}$. Adding $30 \%$ does not improve it anymore since the contribution of the SFE16 starts to dominate.

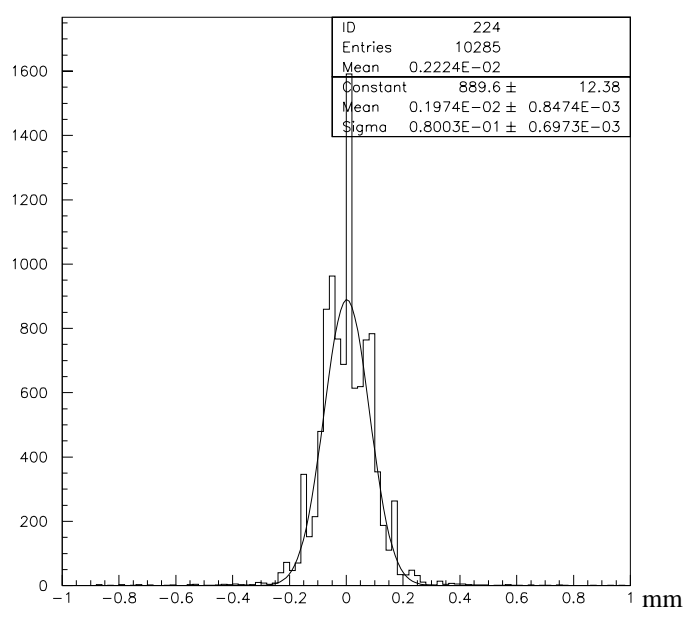

Fig. 4. Spatial resolution measurement. Distribution of residuals between the position of the hit in the detector and the reference track $(\sigma=80 \mu \mathrm{m})$. Contribution from the detector $50 \mu \mathrm{m}$.

The resolution of the large Micromegas equipped with SFE16 was measured using the telescope described in section 4.3. The reference tracks were defined by the hits in 3 other Micromegas of smaller size. For these prototypes equipped with the MQS104 readout the position resolution was estimated to be $85 \mu \mathrm{m}$, resulting in a $65 \mu \mathrm{m}$ resolution for the reference track. The distribution of the residuals of the hit positions in the large Micromegas is shown in the Fig. 4 for the $\mathrm{Ne}-\mathrm{C}_{2} \mathrm{H}_{6}-\mathrm{CF}_{4}$ (79-11-10) mixture. The distribution has 
$\sigma=80 \mu \mathrm{m}$. When subtracting quadratically, we obtain a resolution of $47 \mu \mathrm{m}$ for the large Micromegas. As mentionned in 3.3 the TOT value is used as a weighting factor for each strip of a cluster, in order to improve the resolution.

Smaller mean cluster sizes can be interpreted as less transverse diffusion in the drift space. This translates into a higher precision in the hit position. However, we observed that a better resolution is obtained from events with a cluster size of 2 strips rather than 1 strip. Indeed, the best resolution $(50 \mu \mathrm{m})$ is obtained for the last two gas mixtures shown in Table 1 for which the mean cluster sizes are 2.1 and 2.2 respectively.

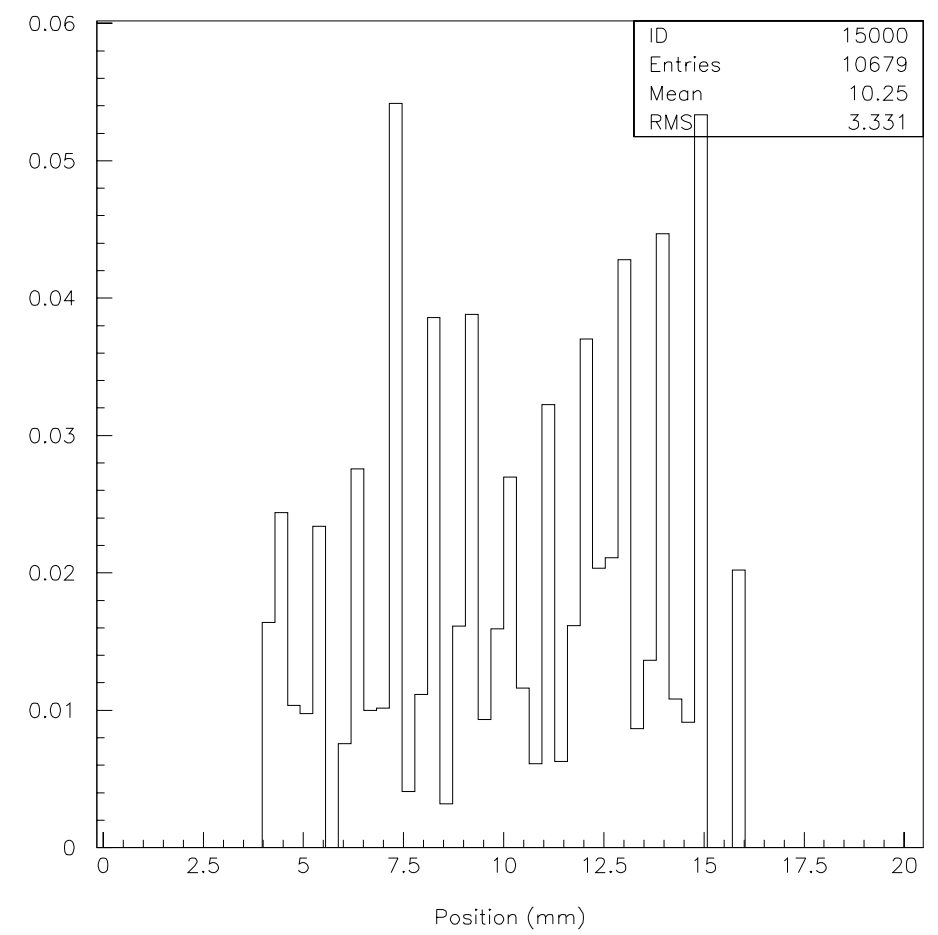

Fig. 5. Inefficiency versus position (one bin per strip). Gas mixture: $\mathrm{Ne}-\mathrm{C}_{2} \mathrm{H}_{6}-\mathrm{CF}_{4}$ (79-11-10); Gain 3800 .

The inefficiency measured as a function of the position (or strip number) in the detector is shown in Fig.5 (Neon mixture, $\mathrm{G}=3800, \epsilon=98 \%$ ). One bin in the histogram corresponds to a single strip in the detector. Every third strip the inefficiency is higher. This can be attributed to the presence of the spacers between the mesh and the board. The spacers hide about $10 \%$ of the surface of these strips ( $2 \%$ of the total active area of the detector) and induce a local inefficiency. This shifts towards higher value the gain $G_{0}$ at which an efficiency of $99 \%$ is reached. The effect is enhanced for gases with low ionization and low transverse diffusion. For instance, with the Neon mixture, efficiencies of 97,98 and $99 \%$ are reached at gains of 2900,3800 and 6400 respectively.

Several criteria will guide us in the final selection of the optimum gas mixture. 
The position resolution translates directly into momentum resolution for the spectrometer, it is therefore a critical parameter. The presence of a high flux of background tracks calls for the best possible timing resolution which will help in reducing the number of tracks having random timing with respect to the trigger. This high flux generates an occupancy in each readout channel which is proportional to the TOT value. Therefore, using a mixture which guarantees full efficiency and a fast collection of the charge (small TOT) is preferable. A reduced cluster size is better for the spatial resolution. For the final choice the frequency of discharge will also be a critical parameter. Finally, non-flammable mixtures will be preferred as well as the cheapest ones.

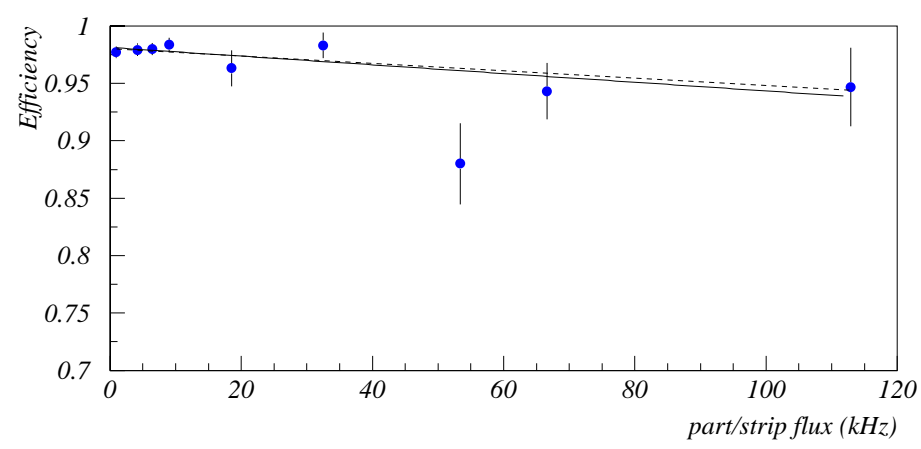

Fig. 6. Efficiency versus flux in a $10 \mathrm{GeV}$ mixed hadron beam. The full line is a fit of the data; the dashed line is the efficiency behaviour expected from the occupancy of the electronics.

\section{$5 \quad$ Efficiency versus flux}

Measurements of efficiency have been performed in the $10 \mathrm{GeV} \pi^{+}$and proton T9 beam. The flux of particles varied from $1 \mathrm{kHz}$ to $120 \mathrm{kHz}$ per strip (length $26 \mathrm{~cm}$, pitch $0.317 \mathrm{~mm}$ ) and was estimated from the random hits in the detector in a 100 ns time gate. The hit rate per strip is $\approx 2$ times the particle rate since the average cluster size is $\approx 2$. Data were taken with the Ne-C2H6-CF4 (79-11-10) mixture, at meah voltages of 430-440 V.

The efficiency was measured using the telescope made of 3 Micromegas of smaller size described before. The data have been corrected for the dead time effect of at most few \% resulting from discharges. Results are shown in Fig.6. The observed drop of efficiency with increasing flux can be attributed to the occupancy of the electronics. The full line is a fit of the data. Due to the limited statistics at high flux, the error on the slope of the fitted line is 100 $\%$. The dashed line is the inefficiency expected from occupancy:

$$
\epsilon=\epsilon_{\max }-2 \times T O T \times \phi
$$


where TOT is the signal width (Time Over Threshold $=170 \mathrm{~ns}$ ), and $\phi$ the particle rate per strip. The factor 2 comes from the requirement of recording both the leading and trailing edges of the signal. Both curves agree which shows that within our errors the observed inefficiency is entirely due to occupancy. It indicates that no drop of gain occurs at high flux. This is confirmed by the fact that the mesh current remains proportional to the flux up to the highest flux value $\left(1.210^{5}\right.$ particles/strip/s $)$.

\section{Discharges studies}

\subsection{Observation of discharges}

Similarly to other micro-strip (or micro-pattern) gas detectors, Micromegas is subject to discharges. It is seen as an abnormal increase of the mesh current which occurs at a low rate compared to the rate of incident particles. The total charge released in a discharge can reach the $\mu \mathrm{C}$ range, even when operating at a gas amplification factor of a few thousands. This indicates that a highly nonlinear amplification process occurs. Contrary to certain other types of microstrip detectors, discharges do not appear to alter permanently the properties of Micromegas. However they require to set up a special protection for the frontend electronics. They also generate dead-time and occupancy; consequently, their rate ought to be minimized. A likely explanation is that a discharge is triggered when an energy of a few hundred $\mathrm{keV}$ is released in the detector drift space resulting in a large number of primary ions. The total charge in the avalanche reaches a limit [7] beyond which amplification diverges. Locally, the gas stops behaving as a dielectric and starts conducting. Contrary to a detector with wires, amplification takes place in a restricted volume with uniform field for such detector with parallel plate structure. This reduces the space-charge effects and allows to operate under high flux. A counterpart seems to be that amplification remains constant for very large primary ionizations, leading to discharge.

For the COMPASS setup, Micromegas is optimized to detect efficiently Minimum Ionizing Particles (MIP). For such particles, even including the tail of the distribution of energy loss (Landau distribution), it is unlikely that the energy deposit exceeds $10 \mathrm{keV}$. This is not the case for particles like secondary particles or nuclear fragments which can deposit much larger amounts of energy. Such candidates may be present in the incoming flux or be produced within the gas detector. It has been shown during our tests that hadrons produce more discharges than electrons or muons. Also, in a given setup, the rate of discharges depends on the detector gain and on the gas mixture. In order to minimize the rate and the impact of discharges, we have tested Micromegas 
detectors filled with different gas mixtures using beams of different particle compositions. For that purpose a special tagging circuit allowed us to trigger on discharges and to study their impact on detection efficiency.

\section{D Polarization of the strips. Dead time associated to discharges}

The rapid change in the current $i_{M}$ drawn by the mesh, which can reach the $\mu \mathrm{A}$ range, indicates that the discharge occurs in the amplification gap (Fig. 7). For some discharges, a local light emission may be observed as well as a characteristic "sparking" noise. For the strips near the discharge the potential gets close to the mesh potential and the gas has become highly conductive. This is expected to reduce amplification and to end the discharge. The change in $i_{M}$ can be integrated, leading to the total charge $\Delta q_{M}$ involved in the discharge.

The present polarization of the strips through individual capacitors as shown in Fig.7 was chosen to reduce the total capacitance and thereby the charge $\Delta q_{M}$ involved in a discharge. It allows the potential of the strips to vary faster than the mesh potential. On the few strips where the discharge takes place, a fast decrease of the potential ( $\sim 400 \mathrm{~V}$ in $25 \mathrm{~ns}$, with $1 \mu$ s recovery time) occurs whereas the change in the mesh potential is much reduced. The resulting large drop in the gain should only affect locally the detector. However, even of a reduced amplitude, the change in the mesh potential can still produce some gain variation and more importantly, it induces a parasitic signal on all the strips which inhibits the highly sensitive FE electronics. This perturbation could not be suppressed, even with the addition of a high capacitance on the mesh.

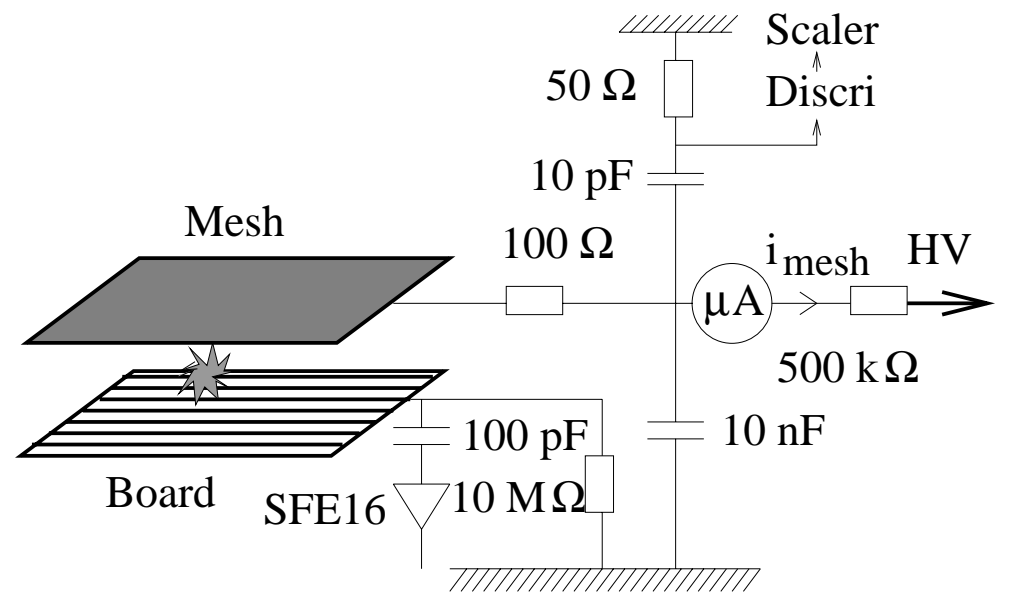

Fig. 7. Schematic view of the circuit for the tagging of discharges

Discharges can be tagged by measuring the change in mesh potential via a capacitor as shown if Fig 7 . The discriminator threshold is set to guarantee 
that every abnormal increase of the mesh current (i.e. discharge) is detected. The precise timing of discharges is therefore known, and they are recorded by scalers. Measurements of the rates of discharges have been performed at CERN using different gas mixtures, both on the T9 $15 \mathrm{GeV}$ secondary hadrons PS beam, and on the M2 $190 \mathrm{GeV}$ SPS COMPASS muon beam.

With the tagging, the efficiency of the detector could be measured as a function of time. Following a discharge it drops to zero which confirms that all the strips are occupied. After about $2 \mathrm{~ms}$ the efficiency recovers a value of $\approx 80 \%$. To get back to $\approx 100 \%$ efficiency requires another $10 \mathrm{~ms}$. This duration is in agreement with a direct observation of the parasitic signal on the mesh and all the strips. The overall mean dead time associated to a discharge was measured to be $3.2 \mathrm{~ms}$.

\subsection{Discharges in Micromegas in a high energy hadron beam}

Measurements of the rates of discharges in Micromegas were performed on the T9 hadron beam at CERN described in 4.3. Data were taken at incident beam flux varying between 0.1 and $2 \mathrm{MHz}$. It has been observed that the rate of discharges, when normalized to the value of the incident flux, does not depend of this value. Therefore, the results are presented as probabilities of a discharge per incident particle. Fig. 8 shows the probablities as a function of the gain for various gas mixtures. Several observations can be made:

(1) Given a gas mixture, the probability of discharge is a power function of the gain.

(2) The probability depends strongly on the mean atomic number $\langle Z\rangle$ or the mean weight $\langle A\rangle$ of the gas mixture.

Indeed, the data points obtained with mixtures having the same values of $<$ $Z>$ values are superimposed. The first set of data, which leads to the highest discharge probabilities (at a given gain), is obtained with $\mathrm{Ar}-i \mathrm{C}_{4} \mathrm{H}_{10}(89-11)$ and $(96-4)$ (percentages) mixtures, and a $N e-C_{2} H_{6}-C F_{4}(59-11-30)$ mixture with $\langle Z\rangle$ values of $19.7,18.6$ and 20.3 respectively. The second set of data is obtained with a $\mathrm{Ne}-\mathrm{C}_{2} \mathrm{H}_{6}-\mathrm{CF}_{4}(79-11-10)$ mixture with $\langle Z\rangle=$ 14. The last set of data which corresponds to the lowest discharge probabilities is obtained with the lightest (tested) mixtures $\mathrm{Ne}-\mathrm{C}_{2} H_{6}(95-5)$ and $(89-11)$ with $\langle Z\rangle=10.4$ and 10.9 , respectively. This observed dependence of the discharge probability with $Z$ (or $A$ ) is by far larger than the one which is seen for the primary ionization produced by MIPs. Such strong dependence of the discharge probability with the gas mixture was previously observed when generating discharges with $5.4 \mathrm{MeV}$ alpha particles from a ${ }^{241} \mathrm{Am}$ radioactive source. The dependence was qualitatively similar although stronger than with 


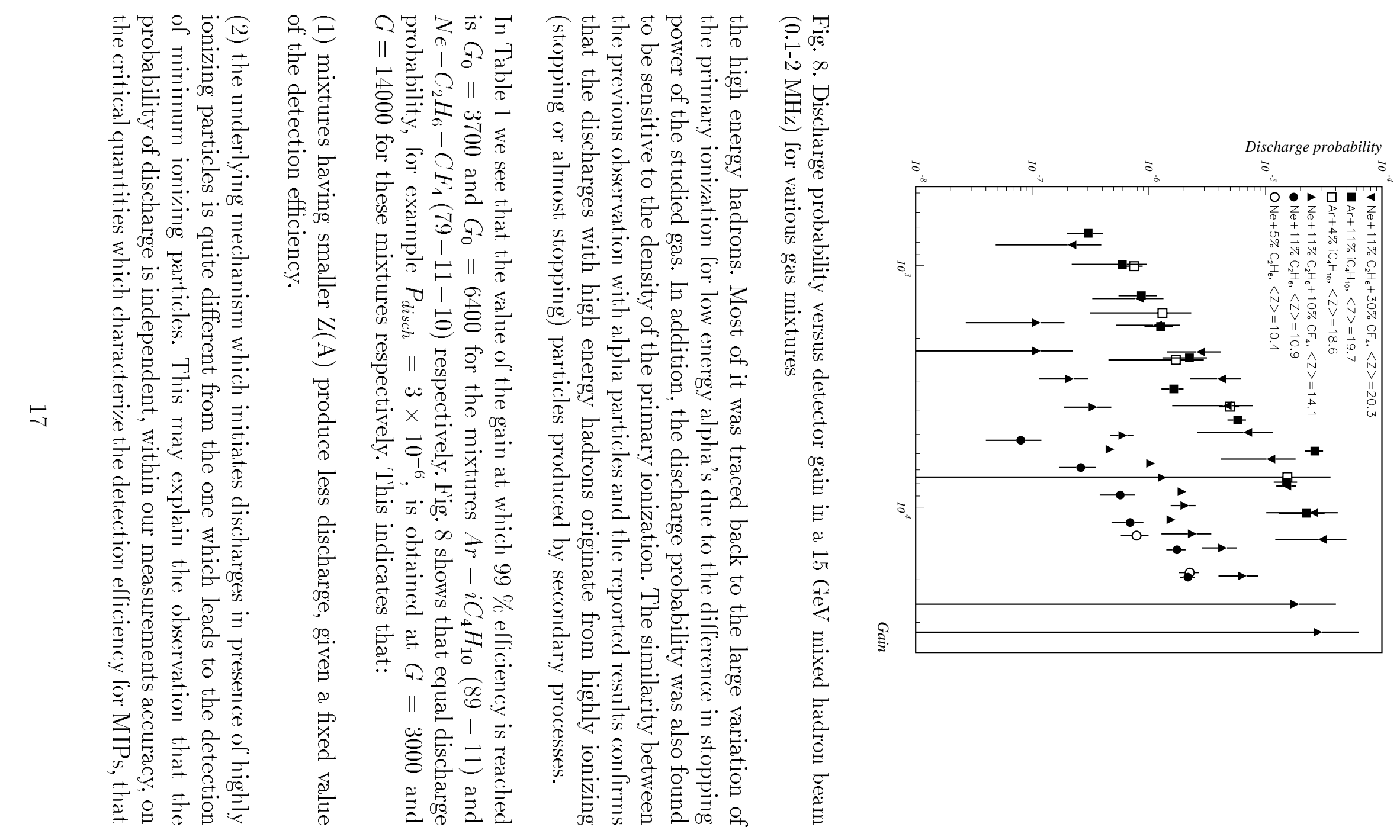


is the drift velocity of electrons and ions and the size of the transverse diffusion. These parameters can therefore be tuned conveniently by an optimum choice of gas mixture, the rate of discharges being given almost exactly by the average Z.

Finally we have also observed that for hadrons energies between 3 and $15 \mathrm{GeV}$, the discharge probabilities are rather insensitive to the energy of the incident particles.

\subsection{Particles flux and discharges rates in the conditions of the COMPASS experiment}

In the COMPASS experiment, Micromegas is foreseen to perform the tracking of charged particles in the vicinity of the high energy M2 muon beam downstream of the fixed (polarized) target. For the (muon) COMPASS program, this beam is designed to have, close to the target, approximately gaussian vertical and horizontal distributions with $\sigma \approx 8 \mathrm{~mm}$. The complex transport system is known to generate a so-called muon-beam halo having degraded energy and phase space which extends beyond the beam axis. The fraction of halo, outside of the central region depends on the energy and also on the specific tuning of certain beam line elements. Typical values for the integrated halo intensities are $20 \%$ and $10 \%$ of the beam intensity, at muon energies of $100 \mathrm{GeV}$ and $200 \mathrm{GeV}$ respectively [5].

In addition, to this beam halo, an intense flux of neutral and charged particles is generated from the complex interactions of the few hundred $\mathrm{GeV}$ muons within the target. This target halo induces a high occupancy in the detectors which perform the tracking, downstream of the target. It is almost entirely swept away after crossing the field integral of $\approx 1$ Tm of the first analysing magnet in the COMPASS setup which indicates that particles from the target halo have sub- $\mathrm{GeV}$ energies.

The complex nature of the particle flux to which Micromegas is subjected in the COMPASS setup makes it impossible to predict the behaviour of the detector in such conditions and particularly to estimate the rate of discharges. In order to test the performances of the detector in these conditions, a Micromegas detector prototype was installed in the $200 \mathrm{GeV}$ M2 muon beam at CERN at $\approx 1.5 \mathrm{~m}$ downstream of the target. The target consisted of 2 cylinders of polyethylene, $65 \mathrm{~cm}$ long each, amounting to $2.7(2.3)$ radiation (nuclear interaction) length in total which could be easily removed from the beam.

In spite of its potentiality to remain linear at high charged particles flux densities, a severe limitation in the maximum flux which Micromegas can detect 
occurs from the value of the shaping time ( $\geq 100 \mathrm{~ns}$ ) required for the front end amplifier. As explained in 3.1, this is mandatory to guarantee high detection efficiency at gains of few thousands where the rate of discharges remain tolerable. To avoid the resulting high occupancy from the muon beam flux, the detector had to be neutralized on a $5 \mathrm{~cm}$ diameter zone centered on the beam axis.

\subsubsection{Particles rates in Micromegas}

Measurements performed at beam intensities varying from $0.1610^{8}$ to $1.410^{8} \mu$ per spill of $2 \mathrm{~s}$ have been normalized to the maximum expected intensity in COMPASS of $2.10^{8} \mu$ per spill.

Since the detector was not fully equipped with the corresponding read out electronics, an estimate of the total flux seen by the detector could be obtained by integrating the rates measured for the equipped strips with the detector set at different distances from the beam. With the dead zone centered on the beam axis, i.e. excluding the beam we obtain a total flux of $\approx 10 \mathrm{MHz}$, in absence of target, compatible with the halo intensities mentionned above. At the border of the dead zone, i.e. at $2.5 \mathrm{~cm}$ from the beam, a flux density of $2.10^{5}$ particles $/ \mathrm{cm}^{2} / \mathrm{s}$ is observed, and the hottest strip sees $10^{5}$ particles $/ \mathrm{s}$.

Measurements with and without target have allowed us to disentangle the contributions from the beam alone and the additional contribution from target. In the presence of target the total flux reaches a value close to $30 \mathrm{MHz}$. We also observe that a rather intense tail from the beam halo extends slightly beyond the dead zone. This tail is almost entirely responsible for the high rates reported above. At $8 \mathrm{~cm}$ from the beam, the beam halo contribution has become small compared to the target halo which generates a single rate per strip of $\sim 30 \mathrm{kHz}$.

\subsubsection{Discharges rates}

Using the same system of tagging as described previously, the rate of discharges was measured as a function of the detector gain, for two gas mixtures $\mathrm{Ar}-$ $i \mathrm{C}_{4} \mathrm{H}_{10}(89-11)$ and $\mathrm{Ne}-\mathrm{C}_{2} \mathrm{H}_{6}-\mathrm{CF}_{4}(79-11-10)$.

The number of discharges per spill of $210^{8} \mu$ are shown in Fig 9 . Two obvious features are seen $1 /$ the rate increases in presence of target which is expected since the target brings an additional halo component, $2 /$ discharge rates are about one order of magnitude lower with the Neon based mixture compared to the Argon based mixture. This last feature confirms the similar property observed previously with the T9 hadron beam. 
Full efficiency of the detector is reached at a gain of $\approx 3700$ in $\mathrm{Ar}$ and $\approx 6400$ in Ne, corresponding to 1.3 and 2.6 discharge per spill using Ar, and 0.1 and 0.4 discharge per spill using Ne, respectively for the data taken without and with target. These values are shown in Table 2 along with the discharge probabilities obtained with the T9 hadron beam. These measurements confirm the advantage of using a mixture based on Neon in order to reduce the rate of discharges.

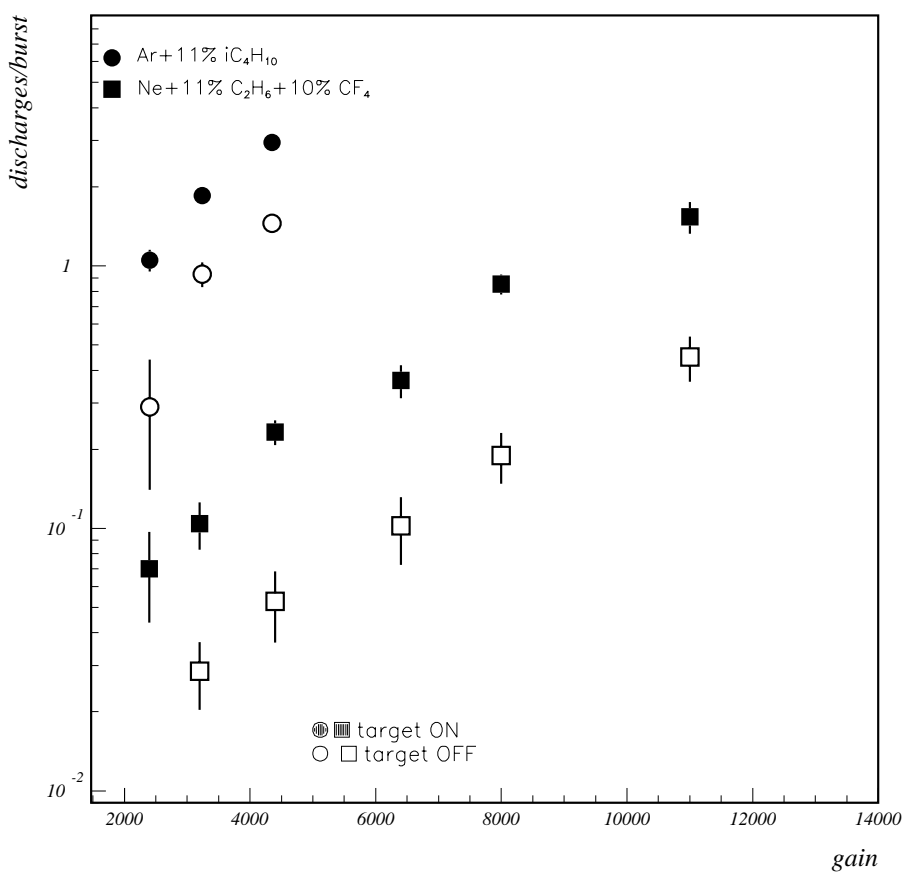

Fig. 9. Number of discharges per spill measured at the gain $G_{0}$ (beginning of the efficiency plateau) in the conditions of the COMPASS experiment. Full circles (squares) correspond to data taken with the target, with the gas mixture based on Argon (Neon). Empty circles and squares correspond to data taken without the target.

Since the flux seen by the detector has different components it was important to check if the muon beam, its associated halo and the additional flux produced by the target generate discharges with comparable probabilities. The detector was shifted a few $\mathrm{cm}$ away from the beam axis, so that the active part of the detector could intercept the whole muon beam plus its halo. The number of discharges did not increase (compared to the number at nominal position with the beam going through the dead zone), in spite of an increase by a factor $\approx 10$ of the incident flux. This indicates that $1 /$ high energy muons generate discharges with probabilities below $10^{-9}$ that is (at least) three orders of magnitude below the probability for hadrons of few $\mathrm{GeV}$ energies, and $2 /$ most of the discharges are generated by particles from the halo. This observation, along with the increase of the number of discharge in presence of target, suggest that the beam halo and target halo contain particles other than high 
Table 2

Probability of discharge per incident particle in the T9 hadron beam $(0.2-1 \mathrm{MHz}$ hadrons), and number of discharges per spill in the M2 muon beam line (2 $10^{8}$ muons) with and without a $1.3 \mathrm{~m}$ polyethylene target. Measurements are performed at the gain $G_{0}$ (beginning of the efficiency plateau)

\begin{tabular}{|c|c|c|c|c|c|c|}
\hline $\begin{array}{l}\text { Gas } \\
\%\end{array}$ & $\begin{array}{l}\mathrm{Ar}_{-} \mathrm{iC}_{4} \mathrm{H}_{10} \\
96-4\end{array}$ & $\begin{array}{l}\mathrm{Ar}-\mathrm{iC}_{4} \mathrm{H}_{10} \\
89-11\end{array}$ & $\begin{array}{l}\mathrm{Ne}-\mathrm{C}_{2} \mathrm{H}_{6} \\
95-5\end{array}$ & $\begin{array}{l}\mathrm{Ne}-\mathrm{C}_{2} \mathrm{H}_{6} \\
89-11\end{array}$ & $\begin{array}{l}\mathrm{NeC}_{2} \mathrm{H}_{6} \mathrm{CF}_{4} \\
79-11-10\end{array}$ & $\begin{array}{l}\mathrm{NeC}_{2} \mathrm{H}_{6} \mathrm{CF}_{4} \\
59-11-30\end{array}$ \\
\hline $\begin{array}{l}\text { Disch. proba.T9 } \\
\text { Disch./spill M2: } \\
\text { With target } \\
\text { Without target }\end{array}$ & $5.10^{-6}$ & $\begin{array}{l}4.10^{-6} \\
2.6 \\
1.3\end{array}$ & $10^{-6}$ & $1.510^{-6}$ & $\begin{array}{l}9.10^{-7} \\
0.4 \\
0.1\end{array}$ & $10^{-5}$ \\
\hline
\end{tabular}

energy muons responsible for the observed discharges.

\section{Conclusions}

A $26 \times 36 \mathrm{~cm}^{2}$ Micromegas microstrip detector with $317 \mu \mathrm{m}$ pitch, was built and tested in a $10-15 \mathrm{GeV}$ hadron beam at CERN. The performances of the detector equipped with the low noise preamplifier SFE16 were measured for various gas mixtures. The best results are obtained with a $\mathrm{Ne}-\mathrm{C}_{2} \mathrm{H}_{6}-$ $C F_{4}$ mixture: $99 \%$ efficiency is obtained at a gain of $\approx 6400$, with a spatial resolution of $50 \mu \mathrm{m}$ and a time jitter of $8.5 \mathrm{~ns}$.

The detector is subject to discharges of current which occur at a rate growing as a power function of the gain, and proportional to the incident flux of hadrons. We have shown that the discharge probability per incident hadron, at a fixed gain, depends on the mean value of the atomic number $\langle Z\rangle$ (or mean atomic mass number $\langle A\rangle$ ), of the gas mixture. For the $\mathrm{Ne}-\mathrm{C}_{2} \mathrm{H}_{6}-\mathrm{CF}_{4}$ mixture, at full efficiency, the probability is smaller than $10^{-6}$.

In the $200 \mathrm{GeV}$ muon beam at CERN, the discharge probability per incident particle is more than three orders of magnitude lower than with hadrons.

We have shown that when operating the detector with the $\mathrm{Ne}-\mathrm{C}_{2} \mathrm{H}_{6}-\mathrm{CF}_{4}$ gas mixture in conditions similar to those of the COMPASS experiment $\left(2.10^{8}\right.$ muons per spill of $2 \mathrm{~s}$, detector with a dead zone of $\phi=5 \mathrm{~cm}$ in the center, downstream of a $1.3 \mathrm{~m}$ long polyethylene target), the rate of discharges is kept at a low rate $(\approx 0.4$ per spill $)$ and produces a negligible dead time.

Such a detector fulfills the requirements for the tracking at small angle in the 
COMPASS experiment.

\section{References}

[1] COMPASS Proposal, CERN/SPSLC/P297, March 1, 1996

[2] Y. Giomataris,Ph. Rebourgeard,J.P. Robert and G. Charpak, Nucl. Instrum. Methods A 376 (1996) 29

[3] D. Thers et al., Nucl. Phys. A 654 (1999) 1037c

[4] E. Delagnes et al., to be published in IEEE Transactions on Nuclear Science

[5] A. Magnon et al., COMPASS note 1998

[6] A. Sharma SLAC-J-ICFA-16-3, July 1998; ICFA Instr. Bull., Vol 16. Nucl. Instrum. Methods A 424 (1999) 321

[7] H. Raether, Z. Phys. 112 (1939) 464 